\title{
ZUR FRAGE DER VERGÖTTLIGHUNG DES VEZIRS (PA-)RAHOTEP
}

von HARTWIG ALTENMÜLLER

Zu den schon früh nach Europa verschlagenen Denkmälern der Zeit Ramses' II. gehört eine Statue des Vezirs (Pa-)Rahotep, die einst in der Villa Altichiero bei Padua aufgestellt war und die sich heute in der Villa Melzi in Bellagio am Comer See befindet. Die bereits am Ende des I8. Jahrhunderts in vier Ansichten veröffentlichte Figur ${ }^{1}$ galt seit langem als verschollen. H. Brunner ${ }^{2}$ hat sie wieder entdeckt und ihr in der Festschrift für J. Cerný einen eingehenden und grundlegenden Aufsatz gewidmet.

Die Figur zeigt den Vezir (Pa-)Rahotep als Würfelhocker. Alle vier Seiten des Hockers waren ursprünglich mit Darstellungen und Inschriften dekoriert. Doch nur die Texte der Vorder- und Rückseite sind heute noch lesbar. Die in einer senkrechten Zeile zwischen den Bildern der Götter Ptah und Osiris entlang geführte Inschrift der Vorderseite nennt den Namen des Parahotep, Bürgermeisters und Vezirs von PiRamses. Die Inschriften der Rückseite, die aus vier senkrechten Zeilen bestehen, setzen an das Ende der jeweiligen Zeilen nach einer längeren Ehrentitulatur den Namen des Vezirs in der artikellosen Form Rahotep, zweimal in Klarschrift und zweimal in änigmatischer Schrift. J. Cerný, ${ }^{3} \mathrm{H}$. de Meulenaere, ${ }^{4}$ und H. Brunner ${ }^{5}$ haben gezeigt, daß Parahotep und Rahotep verschiedene Namensformen eines gleichen Personennamens sein können, sodaß die Annahme berechtigt ist, da $ß$ die auf der Figur festgestellten Namen Parahotep und Rahotep trotz der unterschiedlichen Namensform den gleichen Vezir bezeichnen.

H. de Meulenaere ${ }^{6}$ hat nun nachgewiesen, daß unter Ramses II. zwei Vezire mit dem Namen (Pa-)Rahotep lebten. Der frühere der beiden Vezire stammte aus Abydos. Dort war sein Bruder Wenennefer Hoherpriester des Osiris. Der jüngere kam aus Sedment, dem alten Herakleopolis, wo auch sein Grab gefunden worden ist und wo seine Angehörigen am Tempel des Harsaphes Dienst taten. Es stellte sich daher die Frage, welchem der beiden Vezire der Würfelhocker des (Pa-)Rahotep zugewiesen werden sollte. Dieses Problem schien sehr rasch gelöst. Denn der Vezir (Pa-)Rahotep wurde auf der Vorderseite des Würfelhockers als 'Bürgermeister und Vezir von PiRamses' näher bezeichnet und wies damit einen Amtstitel auf, der auch für den Vezir aus Herakleopolis gut belegt war. Einer Zuweisung der Statue zu den Denkmälern des Vezirs aus Herakleopolis schien nichts mehr im Wege zu stehen. ${ }^{7}$

Trotz des scheinbar klaren Sachverhaltes sind nun aber einige Überlegungen berechtigt, die sich aus der Unterschiedlichkeit der auf der Stele belegten Namens-

I Mad. J. W. C. D(e) R(osenberg), Altichiero (Padua, I788), Taf. I3-I6.

${ }^{2} \mathcal{F E A}{ }_{54}$ (1968), 129-34.

3 Bi. Or. 19 ( 1962$)$, $142 \mathrm{f}$.

4 Chron. d'Ég. 4r (I966), 223.

5 a.a.O., r32 Anm. 4 . 6 a.a.O., $223 \mathrm{ff}$.

7 Ebd. 23I: doc. d; H. Brunner, a.a.O., r32. 
formen sowie aus der Verschiedenheit der für die jeweiligen Vezire genannten Ämter ergeben. Ungewöhnlich scheint zu sein, daß auf einem gleichen Monument der Name des gleichen Vezirs sowohl in der Form Parahotep, als auch in der Form Rahotep begegnet, und sonderbar ist es, daß der Vezir mit der Namensform Parahotep als Vezir von Pi-Ramses erscheint, der Vezir mit der Namensform Rahotep aber nicht mit einem bestimmten Amtssitz, sondern ausschließlich mit religiösen, und zwar speziell memphitischen Priesterämtern verbunden wird. Diese Feststellung ist insofern bemerkenswert, als beobachtet werden kann, daß die Angaben des Würfelhockers über ein eventuelles memphitisches Priesteramt des Vezirs (Pa-)Rahotep aus Pi-Ramses durchaus singulär sind und daß kein anderes, mit Sicherheit dem in Pi-Ramses wirkenden Vezir (Pa-)Rahotep zugewiesenes Denkmal gleichzeitig einen Amtssitz in Pi-Ramses und ein memphitisches Priesteramt aufzählen. Das memphitische Priesteramt wird nur auf einer einzigen Stele des (Pa-)Rahotep ausführlich belegt. ${ }^{8}$ Dieser $(\mathrm{Pa}-)$ Rahotep ist aber dort nicht 'Bürgermeister und Vezir von Pi-Ramses', sondern 'Bürgermeister und Vezir von Memphis'.

Die Stele von Memphis, die für den Vezir (Pa-)Rahotep sowohl verschiedene memphitische Priesterämter, als auch ein Vezirat in Memphis (inbw) ${ }^{9}$ erwähnt, hat bisher stets als ein Denkmal des Vezirs $(\mathrm{Pa}-)$ Rahotep aus Herakleopolis gegolten. ${ }^{\mathrm{IO}}$ Die sichere Neuzuweisung dieser Stele zu den Denkmälern des Vezirs (Pa-)Rahotep aus Abydos ist aber jetzt durch den Vergleich mit einer Statue des (Pa-)Rahotep aus Saqqâra möglich geworden. Diese Statue, die im Jahre I955 in Saqqâra in der Nekropole des Neuen Reiches in der Nähe des Grabes des Chaemwese gefunden wurde, ${ }^{\text {II }}$ dann verschollen war und 1972 wieder entdeckt worden ist, besitzt Inschriften, die sich eng an die Texte der Stele von Memphis anlehnen. ${ }^{12}$ Ähnlich wie die Inschriften auf der Stele von Memphis setzen diese den Vezir in Verbindung mit memphitischen Gottheiten, und ähnlich wie jene verleihen diese dem Vezir (Pa-)Rahotep den Totentitel 'Osiris'. Für die Stele ist daher mit gutem Grund ebenso wie für die Statue eine Herkunft aus der Nekropole von Saqqâra anzunehmen, wo beide ursprünglich im Grabbereich des $(\mathrm{Pa}-)$ Rahotep aufgestellt gewesen sein könnten. Da aber in Saqqâra nur ( $\mathrm{Pa}$-)Rahotep aus Abydos bestattet worden sein kann, weil das $\mathrm{Grab}$ des $(\mathrm{Pa}-) \mathrm{Ra}-$ hotep aus Herakleopolis bei Sedment gefunden wurde, ${ }^{13}$ kann der auf der Grabstele genannte 'Bürgermeister und Vezir von Memphis' nur mit (Pa-)Rahotep aus Abydos identisch sein.

Wird nun aber das Amt des Vezirs und Hohenpriesters von Memphis mit (Pa-) Rahotep aus Abydos und nicht mit (Pa-)Rahotep aus Herakleopolis verbunden,

8 Brugsch, Thesaurus, $v$, $950 \mathrm{f}$.

9 H. de Meulenaere, a.a.O., 226, versucht []$_{\otimes}^{\circ}$ als Kurznamen von Pi-Ramses zu erklären. Er liest daher die Zeichengruppe $\left[{ }_{\otimes}^{\circ}\right.$ als inbt-'Festung'. Diese Interpretation der Zeichengruppe ist kaum zu halten, vor allem wenn man vergleichbare Kurzschreibungen als eine Bezeichnung von inbw - 'Memphis' (H. Gauthier,

Dict. géog. I [1925], 8I) nachweisen kann.

to H. de Meulenaere, a.a.O., 23I: doc. f.

II J. Ph. Lauer, BSFE 22 (1956), 62 f.; J. Leclant, Or. N.S. 25 (1956), 256.

I2 H. Altenmüller und Ahmed M. Moussa, 'Eine wiederentdeckte Statue des Vezirs Rahotep', in $M D A I K$ 30 (1974), rff.

13 W. M. F. Petrie and G. Brunton, Sedment, II (1924), 28 ff., Taf. $71-6,84$. 
ergeben sich einige Schwierigkeiten bei der zeitlichen Einordnung des Vezirs. Das Hohepriesteramt von Memphis wird nämlich während des größten Teils der Regierung Ramses' II. vom Prinzen Chaemwese bekleidet. Mindestens 37 Jahre lang, vom I6. bis 52. Jahr der Regierung seines Vaters, ist Chaemwese Hohepriester von Memphis gewesen. ${ }^{14}$ Daher kann dieses Amt durch (Pa-)Rahotep aus Abydos höchstens vor dem I6. Jahr oder nach dem 52. Jahr Ramses' II. besetzt worden sein. Um hier zu einer Entscheidung zu kommen, muß das für (Pa-)Rahotep aus Abydos vorliegende prosopographische Material auf seinen zeitlichen Ansatz hin näher untersucht werden. ${ }^{15}$

(Pa-)Rahotep aus Abydos wird auf zwei Denkmälern seines 'Bruders' Wenennefer aus Abydos gemeinsam mit dem 'Bürgermeister und Vezir Nebamun' genannt. ${ }^{16}$ Nebamun, der auf den genannten beiden Denkmälern seinerseits als 'Bruder' des Wenennefer erscheint, dürfte-obwohl er mit Sicherheit weder leiblicher Bruder des (Pa-)Rahotep noch des Wenennefer war $^{17}$ - in engem Verwandtschaftsverhältnis $\mathrm{zu}$ beiden Personen gestanden haben. $\mathrm{Da}$ Nebamun und ( $\mathrm{Pa}-$ )Rahotep aus Abydos beide wiederholt als 'Brüder' des Wenennefer auf einem gleichen Monument nebeneinander erwähnt werden, kann deren Lebenszeit aber nicht allzu weit auseinander gelegen haben. Nebamun war Vezir zur Zeit des Königs Sethos I. ${ }^{18}$ Das Hohepriesteramt des Vezirs (Pa-)Rahotep aus Abydos dürfte daher in der ersten Hälfte der Regierung Ramses' II. liegen. Sollten nun aber beide Vezire, wie es nunmehr erscheint, annähernd gleich alt gewesen sein, kann ( $\mathrm{Pa}$-)Rahotep aus Abydos kaum das 52. Jahr Ramses' II. erreicht haben. Dem widersprechen auch nicht die Angaben einer Stele des Wenennefer aus Abydos, auf der im 42. Jahr Ramses' II. der Name des (Pa-)Rahotep zum letzten $\mathrm{Mal}$ zusammen mit dem seines 'Bruders' Wenennefer erscheint. ${ }^{19}$ (Pa-)Rahotep könnte sehr wohl bereits im I6. Jahr Ramses' II. gestorben sein und wegen einer gewissen Berühmtheit auch noch einige Zeit nach seinem Tode auf dem Denkmal seines leiblichen Bruders genannt worden sein. Denkbar wäre aber auch eine Lebenszeit bis zum 42. Jahr Ramses' II. über das I6. Jahr hinaus, wenn angenommen wird, daß der alternde (Pa-)Rahotep im I6. Jahr der Regierung Ramses' II. den Prinzen Chaemwese als seinen Kollegen in das Hohepriesteramt von Memphis mit aufgenommen hat.

$\mathrm{H}$. de Meulenaere ${ }^{20}$ hat festgestellt, daß (Pa-)Rahotep aus Herakleopolis jünger war als der gleichnamige Vezir aus Abydos. Der zeitliche Ansatz der Vezirs (Pa-)Rahotep aus Abydos an den Beginn der Regierung Ramses' II. erfordert daher einen entsprechend späteren Ansatz des Vezirs (Pa-)Rahotep aus Herakleopolis. Für ihn käme eine Amtszeit in der Mitte oder am Ende der Regierung Ramses' II. in Betracht. Ein Ansatz in der späteren Zeit der Regierung Ramses' II. wird dann auch besonders durch die Bezeichnung des Amtssitzes nahegelegt. (Pa-)Rahotep aus Herakleopolis

14 W. Helck, Materialien zur Wirtschaftsgeschichte des Neuen Reiches, I (I960), I32.

15 H. de Meulenaere, a.a.O., 23I f.: doc. o-v.

16 Statue Athen 106: G. Legrain, Rec. trav. 3I (1909), 202 f.; Gruppe des Museums Kairo: Ebd., 204 ff.

I7 H. Kees, Das Priestertum im ägyptischen Staat (I953), I02.

I8 Statue Kairo, CG II40: L. Borchardt, Statuen und Statuetten, IV (I934), 70; vgl. W. Spiegelberg, Rechnungen aus der Zeit Setis I. (1896), Taf. 16 b, a. 2.

I9 Kairo, JE 34505: A. Mariette, Abydos, II (r880), Taf. 4I.

20 a.a.O., 230 
amtierte in Pi-Ramses. Einmal erscheint dieser Amtssitz in der späten Form $p^{3}$ $R r-m s s p_{3} k_{3} n p_{3} R r{ }^{21}$ Diese Form des Ortsnamens von Pi-Ramses ist erst am Ende der Regierung Ramses' II. gebräuchlich und kann zum ersten Mal im 52. Jahr Ramses' II. belegt werden. ${ }^{22}$

Unter dem Vezirat des (Pa-)Rahotep aus Herakleopolis in der zweiten Hälfte der Regierung Ramses' II. und vermutlich sogleich nach dem Tod des Chaemwese im 52. Jahr Ramses' II. ist Pahemnetjer, der Vater des Vezirs (Pa-)Rahotep aus Herakleopolis, ${ }^{23}$ in die freigewordene Stelle des Hohenpriesters von Memphis eingewiesen worden. Auf diesen dürfte Didia gefolgt sein, wenn nicht angenommen werden will, daß dieser gleichzeitig und kollegial mit Pahemnetjer amtiert hat. ${ }^{24}$ Didia war Bruder des (Pa-)Rahotep aus Herakleopolis ${ }^{25}$ oder dessen Schwiegervater ${ }^{26}$ und wird auf einer Stele des Pahemnetjer gemeinsam mit Pahemnetjer, als Hoherpriester von Memphis geführt. ${ }^{27}$

Werden nun aber Pahemnetjer und Didia als Nachfolger des Chaemwese im Hohepriesteramt von Memphis betrachtet, erscheint es ausgeschlossen, daß ( $\mathrm{Pa}-$ ) Rahotep aus Herakleopolis jemals selbst das Hohepriesteramt von Memphis übernommen hat. Denn gleichzeitig mit dem in Pi-Ramses wirkenden (Pa-)Rahotep aus Herakleopolis amtierte in Theben der Vezir Neferrenpet. ${ }^{28}$ Dieser Vezir aus Theben verkündete im 57. und 6o. Jahr Ramses' II. das Io. und II. Sedfest des Königs und muß spätestens nach Didia das Amt des Hohenpriesters von Memphis übernommen haben. Wahrscheinlich hat er es als 'Pensionierung' erhalten. W. Helck nimmt an, daß diese 'Pensionierung' nicht erst unter Merenptah, sondern bereits am Ende der 66-jährigen Regierung Ramses' II. erfolgt ist, ${ }^{29}$ da Denkmäler des Neferrenpet, die sein Hohepriesteramt von Memphis erwähnen, noch unter Ramses II. datiert sind. ${ }^{30}$ Die jeweilige Amtszeit der beiden Hohenpriester Pahemnetjer und Didia kann daher unter diesen Umständen nur sehr kurz gedauert haben. Für ein Hohepriesteramt des $(\mathrm{Pa}-)$ Rahotep aus Herakleopolis aber ist dann aus zeitlichen Gründen nicht mehr genügend Platz.

Wird das Amt des Hohenpriesters von Memphis nunmehr endgültig mit dem Vezir $(\mathrm{Pa}-)$ Rahotep aus Abydos und nicht mit $(\mathrm{Pa}-)$ Rahotep aus Herakleopolis verbunden, ist es unwahrscheinlich, daß der Würfelhocker der Villa Melzi in Bellagio, der als einziges Monument für den Vezir ( $\mathrm{Pa}$-) Rahotep - und dies bemerkenswerterweise unter den verschiedenen Namensformen Parahotep und Rahotep - sowohl ein Vezirat in

${ }_{21}$ A. Mariette, Catalogue des monuments d'Abydos (1880), 424 f. Nr. II 38.

22 Mahmud Hamza, $A S A E$ 30 (1930), 44.

${ }_{23}$ Brit. Mus. 712: T. G. H. James, Hieroglyphic Texts from Egyptian Stelae, 9 (1970), Taf. I4.

${ }^{24}$ Eine kollegiale Verwaltung des Hohepriesteramtes könnte durch die gemeinsame Nennung der beiden Hohenpriester auf der Stele Brit. Mus. I83 nahe gelegt werden.

25 H. de Meulenaere, a.a.O., 227.

26 H. Kees, a.a.O., 103.

27 Brit. Mus. 183: T. G. H. James, a.a.O., 9 (1970), Taf. 15.

28 W. Helck, Zur Verwaltung des Mittleren und Neuen Reiches (1958), $322 \mathrm{ff} ., 45 \mathrm{If}$.

29 Ebd., 324.

30 Berlin 2290: H. Schäfer und G. Roeder, Ägyptische Inschriften aus den königlichen Museen zu Berlin, II (1924), $316 \mathrm{f}$. 
Pi-Ramses, als auch das Hohepriesteramt von Memphis belegen könnte, dem Vezir (Pa-)Rahotep aus Herakleopolis zugeteilt wird. Auf Grund der oben angestellten Überlegungen und Berechnungen erscheint es nämlich ausgeschlossen, daß (Pa-)Rahotep aus Herakleopolis, für den das Vezirat in Pi-Ramses sicher belegt ist, jemals das Hohepriesteramt von Memphis bekleidet hat. Daher sind die in den Inschriften des Würfelhockers genannten beiden Namen Parahotep und Rahotep auf zwei verschiedene Vezire zu verteilen. Die Inschriftzeile der Vorderseite, die ein Vezirat in Pi-Ramses erwähnt, müßte dabei dem Vezir ( $\mathrm{Pa}-)$ Rahotep aus Herakleopolis zugewiesen werden, die vierzeilige Inschrift der Rückseite, die auf das memphitische Hohepriesteramt anspielt, dem Vezir (Pa-)Rahotep aus Abydos. In dieser Inschrift wird der Name des

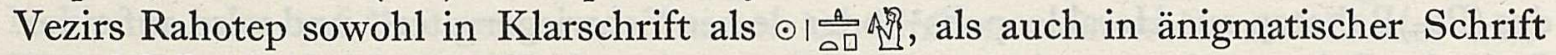

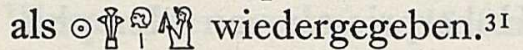

Unter der Voraussetzung, daß der Würfelhocker der Villa Melzi zwei verschiedene Vezire des Namens ( $\mathrm{Pa}-$-)Rahotep nennt, muß versucht werden, das gegenseitige Verhältnis der den verschiedenen Veziren zugewiesenen Inschriften zu definieren. Da dabei die auf der Vorderseite des Würfelhockers eingravierte einzeilige Inschrift des $(\mathrm{Pa}-)$ Rahotep aus Herakleopolis einer vierzeiligen Inschrift des (Pa-)Rahotep aus Abydos auf der Rückseite gegenüber gestellt wird, ist es naheliegend, die einzeilige Inschrift der Vorderseite als Widmungsinschrift des ( $\mathrm{Pa}-$ )Rahotep aus Herakleopolis für den älteren (Pa-)Rahotep aus Abydos aufzufassen und damit die Figur selbst als Weihefigur des (Pa-)Rahotep aus Herakleopolis an seinen bedeutenden Vorgänger zu interpretieren. Die Deutung des Würfelhockers als einer von (Pa-)Rahotep gestifteten Figur wäre denkbar, müßte aber, um voll zu überzeugen, noch durch weitere Inschriften abgesichert werden. Dies ist jedoch gegenwärtig leider nicht möglich, da die Texte der beiden Seiten des Würfelhockers, die Reste von Opfergebeten enthalten, ${ }^{32}$ wegen allzu großer Zerstörung nicht mehr lesbar sind.

Trotz des unter den gegebenen Umständen noch hypothetischen Charakters einer Interpretation des Würfelhockers der Villa Melzi als einer möglichen Weihefigur des $(\mathrm{Pa}-)$ Rahotep aus Herakleopolis an seinen Vorgänger $(\mathrm{Pa}-)$ Rahotep aus Abydos läßt sich die hier vorgeschlagene Deutung weiter erhärten. Wertvolle Hilfe leistet dabei eine Felsinschrift des Wadi Hammamat aus der Zeit Darius' I., in der Chnumibre, der Oberbaumeister des Großkönigs, seine verschiedenen Vorfahren katalogartig aufzählt. An die Spitze seiner Ahnen setzt er dabei den 'Priester des Amun-Re, des Königs der Götter, den Herrn der Geheimnisse des Hauses des Re, den Oberbaumeister von Ober- und Unterägypten, den Bürgermeister und Vezir Rahotep, dessen Ruf über den des Oberbaumeisters von Ober- und Unterägypten, des Bürgermeisters und Vezirs, des obersten Vorlesepriesters des Königs von Ober- und Unterägypten Djoser, (nämlich) des Imhotep geht, des Sohnes des Oberbaumeisters von Ober- und Unterägypten Kanefer, der von der Hausherrin Satnefertum geboren worden ist'. ${ }^{33}$

${ }^{31}$ H. Brunner, a.a.O., I32. Brunner macht a.a.O., Anm. 6 darauf aufmerksam, daß gerade der Vezir aus Abydos Schriftspielereien liebt und führt als Beleg den Würfelhocker Boston, MFA 03. I891 an.

32 H. Brunner, a.a.O., r3o f.

33 J. Couyat and P. Montet, Inscriptions hiéroglyphiques et hiératiques du Ouâdi Hammâmât (r9r2), Taf. 22; G. Posener, Première Domination perse en Égypte (1936), 99 ff. : doc. 14, 26 ff. 
Für den Oberbaumeister Chnumibre liegt die Vergleichbarkeit zwischen den beiden berühmten Persönlichkeiten ( $\mathrm{Pa}$-)Rahotep und Imhotep, von denen zumindest der Vezir (Pa-)Rahotep als möglicher Ahnherr des Chnumibre in Betracht kommt, ${ }^{34}$ in deren beider überragenden Tätigkeit im Bauwesen. Einen solchen Wirkungsbereich erwähnt (Pa-)Rahotep aus Abydos an zwei Stellen. In der Inschrift seines Würfelhockers aus Abydos sagt er von sich selbst: 'Mein Herr machte mich zum Vezir, um den Ptahtempel entstehen zu lassen.'35 Auf der dem (Pa-)Rahotep aus Abydos zugeschriebenen Stele aus Memphis trägt er nicht nur die Titel der Hohenpriester von Heliopolis und Memphis, sondern auch den eines 'Oberbaumeisters' (imj-r3 $k 3 w t) .{ }^{36}$

Eine bis an die Vergöttlichung reichende Verehrung von Baumeistern im Alten Ägypten ist nichts Außergewöhnliches. Sowohl Imhotep aus der Zeit des Djoser, als auch Amenophis Sohn des Hapu aus der Zeit Amenophis' III. haben ihren Ruhm für die Nachwelt vor allem durch die von ihnen erstellten Bauwerke gesichert. Imhotep gilt in den Augen der Nachwelt als der 'Erfinder des Steinbaues' schlechthin. ${ }^{37} \mathrm{Zu}$ den größten Leistungen seiner Bautätigkeit gehört die noch heute bewundernswerte Grabanlage des Königs Djoser in Saqqâra. Ähnliches ist bei Amenophis Sohn des Hapu $\mathrm{zu}$ beobachten, der für Amenophis III. mehrere Tempelbauten errichtet hat und darüber auch in seiner Biographie an verschiedenen Stellen spricht. ${ }^{38}$ Warum sollte daher nicht auch der Vezir (Pa-)Rahotep aus Abydos aus ähnlichen Gründen von der Nachwelt verehrt worden sein? Ist er doch nach eigenen Angaben der Vezir unter Ramses II., der den Ptahtempel von Memphis umgebaut und erweitert hat. Die Ruinen des von ihm, möglicherweise in Gemeinschaftsarbeit mit Chaemwese errichteten Bauwerkes vermitteln noch heute einen Eindruck von der ursprünglichen Monumentalität des damals geschaffenen Tempels.

Es wäre schön, wenn die besondere Leistung erkennbar wäre, die zur Verehrung des (Pa-)Rahotep in späterer Zeit geführt hat. Doch sind in dieser Frage nur hypothetische Antworten möglich, da die Anonymität des Künstlers zum Berufsethos der ägyptischen Handwerker, Künstler und Architekten gehörte und daher keine Nachrichten über die verschiedenen Bauten des ( $\mathrm{Pa}-)$ Rahotep existieren. Erschwerend kommt bei $(\mathrm{Pa}-)$ Rahotep hinzu, daß die eigene Leistung nur ungenau von der seines ebenso berühmten jüngeren Zeitgenossen Chaemwese zu trennen sein dürfte. Dennoch sei hier eine Vermutung geäußert, die in das Bild der Zeit Ramses' II. hineinpassen könnte. Vielleicht liegt die besondere Verehrung des (Pa-)Rahotep aus Abydos, die ihn auch gegenüber Chaemwese abheben dürfte, in der Schaffung der neuen Residenz Pi-Ramses bei Kantir und der Erbauung und Ausschmückung der dortigen Tempelanlagen. Der Namen der unter Ramses II. neugeschaffenen Residenz PiRamses ist zum ersten Mal mit Sicherheit im 8. Jahr Ramses' II. erwähnt, ${ }^{39}$ eventuell

${ }_{34} \mathrm{~K}$. Sethe, Imhotep (UGÄ̈ 2, 1902), 106 f.; vgl. D. Wildung, Die Rolle ägyptischer Könige im Bewußtsein ihrer Nachwelt, I (1969), 83 .

35 Boston, MFA 03. 1891: W. M. F. Petrie, Abydos, II (1903), Taf. 35. 2, 37.

36 Brugsch, Thesaurus, v, 950 .

$37 \mathrm{~K}$. Sethe, a.a.O., II $3 \mathrm{f}$.

38 A. Varille, Inscriptions concernant l'architecte Amenhotep fils de Hapou (1968), 8: Text 2; 27 : Text I r. 3 ff.; 37: Text I3. I6f.; 130 . 
bereits im 5. Jahr dieses Herrschers. ${ }^{40}$ Der Ausbau und die Ausschmückung der neuen Residenz dürfte daher am Beginn der Regierung Ramses' II. erfolgt sein und unter der Aufsicht des Vezirs von Memphis gestanden haben. Als dieser kommt nur (Pa-)Rahotep aus Abydos in Betracht, der den Ausbau der Residenz mit besonderem Nachdruck vorangetrieben haben könnte. Indirekte Belege für seine Aktivität in PiRamses, bei Kantir im Ostdelta, sind die zahlreichen memphitischen Denkmäler aus der Zeit vor Ramses II., die in Tanis gefunden worden sind. ${ }^{4 I}$

Die Annahme einer möglichen aktiven Beteiligung des Vezirs von Memphis (Pa-) Rahotep an der Erbauung und Ausschmückung der Residenz von Pi-Ramses würde sich sehr gut in das bisher von ( $\mathrm{Pa}-)$ Rahotep aus Abydos gewonnene Bild einfügen. Zugleich könnte auch erklärt werden, warum sein Nachfolger ( $\mathrm{Pa}-)$ Rahotep aus Herakleopolis in seiner Funktion als Vezir von Pi-Ramses seinem Vorgänger (Pa-) Rahotep eine Weihefigur stiftete. Denn ( $\mathrm{Pa}-)$ Rahotep aus Herakleopolis scheint der erste Vezir der Zeit Ramses' II. gewesen zu sein, der seinen dauernden Amtssitz nach Pi-Ramses verlegt hat. ${ }^{42} \mathrm{Z}$ war läßt sich der thebanische Vezir Paser, der ein Zeitgenosse des (Pa-)Rahotep aus Abydos gewesen zu sein scheint, bereits im I7. Jahr Ramses' II. in Pi-Ramses nachweisen. ${ }^{33}$ Doch besaß er dort nicht einen Amtssitz, sondern nur ein Wohnhaus. Nach wie vor darf daher angenommen werden, daß (Pa-)Rahotep aus Herakleopolis der erste Vezir von Pi-Ramses war und zur Bekundung seines neuen Amtssitzes, und vielleicht auch, um sich von (Pa-)Rahotep aus Abydos abzuheben, seinen Namen fast regelmäßig mit dem neuen Amtstitel verband: 'Bürgermeister und Vezir von Pi-Ramses'.

40 A. H. Gardiner, $\mathscr{F} E A$ (1918), I79 f.

$4 \mathrm{I}$ J. v. Beckerath, Tanis und Theben (195 I), $4 \mathrm{I}$ ff.

$42 \mathrm{vgl}$. H. de Meulenaere, a.a.O., 225.

43 Labib Habachi, $A S A E 52$ (1952), 480; vgl. auch die Stele aus Tanis (Rec. trav. 22 [1899], I10). 